\title{
Multi-detector CT Evaluation of Liver Neoplasms
}

\author{
Kalpesh K. Patel ${ }^{1}$, Mayur V. Khandhedia ${ }^{2}$, Vishalkumar H. Bhardava ${ }^{3}$ \\ ${ }^{1}$ Assistant Professor, ${ }^{2}$ Associate Professor, ${ }^{3}$ Assistant Professor, Department of Radiology, Dhiraj Hospital, SBKS Medical \\ Institute and Research Centre, Sumandeep Vidhyapeeth University, Pipariya, Vadodara, India
}

Corresponding author: Mayur V. Khandhedia, 2, Kanha Bunglow, Opp. Vrundavan Bunglow, Kubereshwar Marg, WaghodiaDabhoi Ring Road, Vadodara-390019, India

DOI: $10.21276 / \mathrm{ijcmsr}$.2018.3.3.35

How to cite this article: Kalpesh K. Patel, Mayur V. Khandhedia, Vishalkumar H. Bhardava. Multi-detector CT evaluation of liver neoplasms. International Journal of Contemporary Medicine Surgery and Radiology. 2018;3(3):C158-C163.

\section{A B S T R A C T}

Introduction: Introduction of Multi Detector computed Tomography has been a huge leap in the CT technology. MDCT has a high diagnostic accuracy, sensitivity and specificity in the evaluation of focal and diffuse lesions of liver. Current study aimed to study the role of MDCT as a diagnostic modality for the neoplastic lesions of liver, to diagnose and characterize focal liver lesions with help of Multidetector CT scan and to differentiate benign form malignant lesion and primary malignant from metastases.

Material and methods: A prospective study of the role of MDCT in detecting and characterizing neoplastic hepatic lesions was conducted in the period from December, 2015 to December, 2017 in radiology department of Dhiraj Hospital, SBKSMIRC, Sumandeep Vidhyapeeth University, Pipariya, Vadodara. 80 patients, with hepatic neoplastic lesions, were selected. Diagnosis was confirmed by surgery and histopathology, follow up imaging and other non radiological investigations.

Results: Out of 15 cases of Hepatocellular carcinoma 13 (86.67\%) showed heterogeneous hyperenhancement in the arterial phase; 8 (53.33\%) cases of hepatocellular carcinoma were hypoattenuating and 5 (33.33\%) cases were isoattenuating in portal venous phase, suggestive of early washout. Out of 41 cases of metastases 39 (95.12\%) cases of metastases showed hypoattenuation in arterial phase and portal venous phase while, in the venous phase 12 (29.27\%) cases showed hypoattenuation and 27 (65.85\%) cases showed isoattenuation. Out of 65 cases of adults with neoplastic lesions of liver including hepatocellular carcinoma, metastases, hemangioendothelioma, haemangioma and intrahepatic cholangiocarcinoma, sensitivity and specificity of MDCT for hepatocellular carcinoma was $86.7 \%$ and $98 \%$ respectively.

Conclusion: MDCT is "The Imaging Modality of choice" in characterizing hepatic neoplasms and is highly sensitive for detection of hepatocellular carcinoma and hepatic metastases.

Key words: Hepatocellular Carcinoma, Metastases, Hyperenhancement in Arterial Phase, Washout of Contrast, Hypovascular

\section{INTRODUCTION}

CT remains an exciting branch of the radiology, due to several recent technical innovations and improvements. With ever emerging improved techniques, new CT applications continue to be added to an already existing long list of applications. Over the past few years, introduction of Multi Detector Computed Tomography has been a huge leap in the CT technology. CT scanning has become a versatile tool in the evaluation of liver pathologies. MDCT has a high diagnostic accuracy, sensitivity and specificity in the evaluation of focal and diffuse lesions of liver. Triple phase studies of the liver can be performed by timing the data acquisition during late arterial phase, hepatic parenchymal phase and venous phase. MDCT angiography provides a non invasive way of assessment of vascular involvement and feeding vessel supplying focal liver lesion.

Study aimed to study the role of MDCT as a diagnostic modality for the neoplastic lesions of liver, to diagnose and characterize focal liver lesions with help of Multidetector CT scan and to differentiate benign form malignant lesion and primary malignant from metastases.

\section{MATERIAL AND METHODS}

A prospective study of the role of Multidetector CT scan in detecting and characterizing neoplastic lesions of liver was conducted in the period from December, 2015 to December, 2017 in radiology department of Dhiraj Hospital, SBKSMIRC, Sumandeep Vidhyapeeth University, Pipariya, Vadodara, India. 80 patients, who presented with clinical signs and symptoms pertaining to neoplastic processes of liver, were selected for this study. Confirmation of diagnosis was done by following up patients with surgery and histopathology, follow up ultrasonography or Computed Tomography and other non radiological investigations.

\section{Scanning and Methods Used}

CT scan was performed on Siemens 16 slice Multidetector CT scanner machine. Volumetric data from diaphragm to rectum were acquired with pitch kept usually 1 with contiguous $0.625 \mathrm{~mm}$ slice thickness. Reconstruction algorithm used to obtain $1 \mathrm{~mm}$ voxels in axial, coronal and 
sagittal planes and also in multiple planes. Each patient was administered 1 to $1.5 \mathrm{ml} / \mathrm{kg}$ body weight nonionic intravenous contrast (Iohexol $350 \mathrm{mg} / \mathrm{ml}$ ) through power injector at rate of $2.5-3 \mathrm{ml} / \mathrm{sec}$.

Images were acquired after oral and intravenous contrast with triple phase or arterial and venous phase as and when required.

\section{Inclusion criteria}

Patients, clinical presentation suggested neoplastic lesions of liver with CT scan findings suggestive of neoplastic lesions of liver and in whom biopsy and histopathological correlation was done were included.

Patients with primary malignant lesion elsewhere with suspicious metastatic lesions in liver were included.

All patients who could be rescanned with USG/CT for follow up or followed for surgical / histopathological confirmation.

\section{Exclusion criteria}

Patients with infective or inflammatory lesion of liver were excluded.

Patients with traumatic lesions of liver were excluded.

Patients who could not be followed up to the final diagnosis are excluded.

For calculation of sensitivity and specificity of MDCT for diagnosis of hepatocellular carcinoma, 13 cases of simple cysts and 12 cases of hepatoblastoma were excluded.

\section{STATISTICAL ANALYSIS}

All the results were recorded in Microsoft excel sheet and analysed descriptively using statistical formulas for sensitivity and specificity.

\section{RESULTS}

The age of the patients involved in study ranges from new born to $90 \mathrm{yrs}$ old people. The lesions are more common in 41-70 yrs of age group, $62(77.5 \%)$ out of 80 patients. Pathology is more common in male, 45 out of 80 (56.25\%) as compared to females, 35 out of 80 (43.75\%). The most common presentation of patients is weight loss in 56(70\%) patients and anorexia in $53(66.25 \%)$ patients.

\section{Morphological NECT Findings}

Study showed that, Out of 15 cases of histopathologically or follow up imaging proven Hepatocellular carcinoma (HCC), 14 (93.33\%) were large (>3 cm) lesions; 10

\section{No. of patients according to age groups}

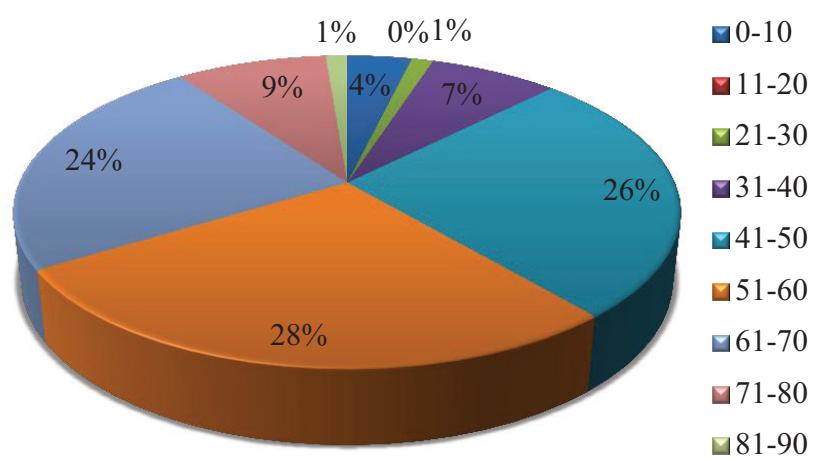

Chart-1: Number of patients according to age group.

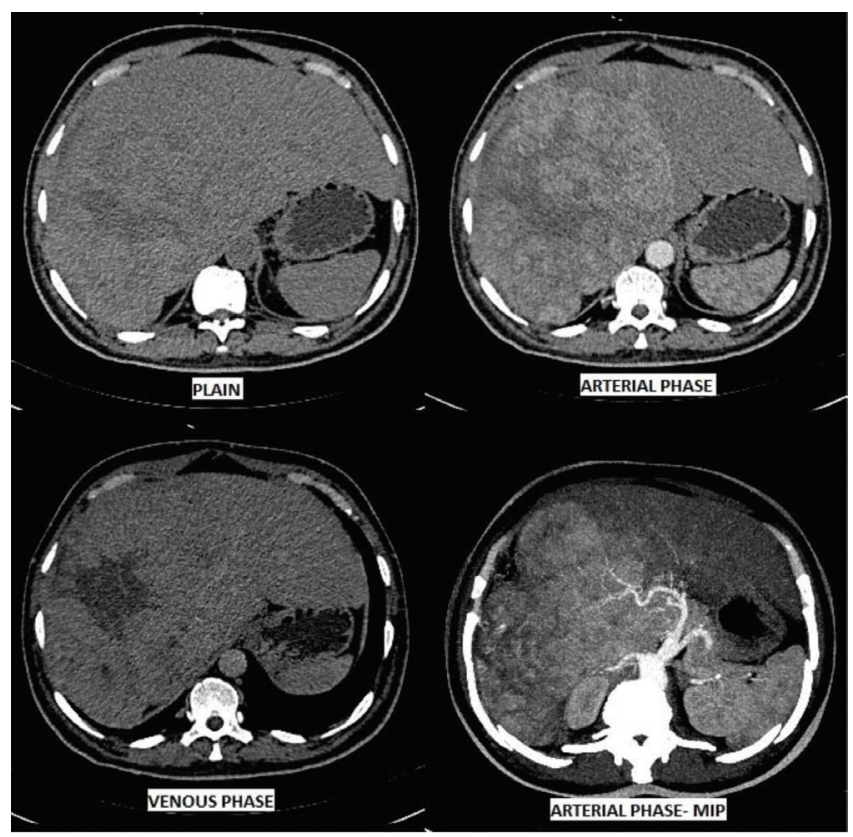

Figure-1: Axial CT scan images though liver in nonenhanced, arterial phase, venous phase with maximum intensity projection (MIP) arterial phase image: hepatocellular carcinoma.

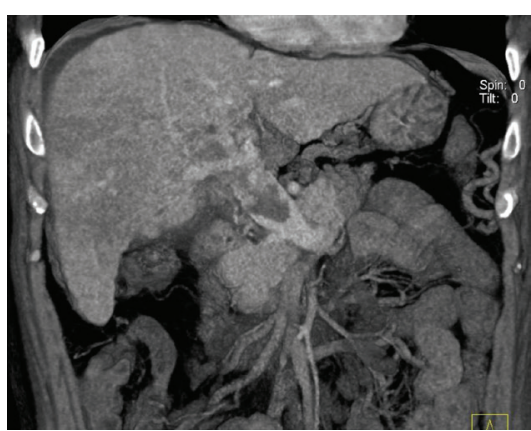

Figure-2: Coronal maximum intensity projection (MIP) CT image in portal venous phase: hepatocellular carcinoma with tumour thrombus in portal vein.

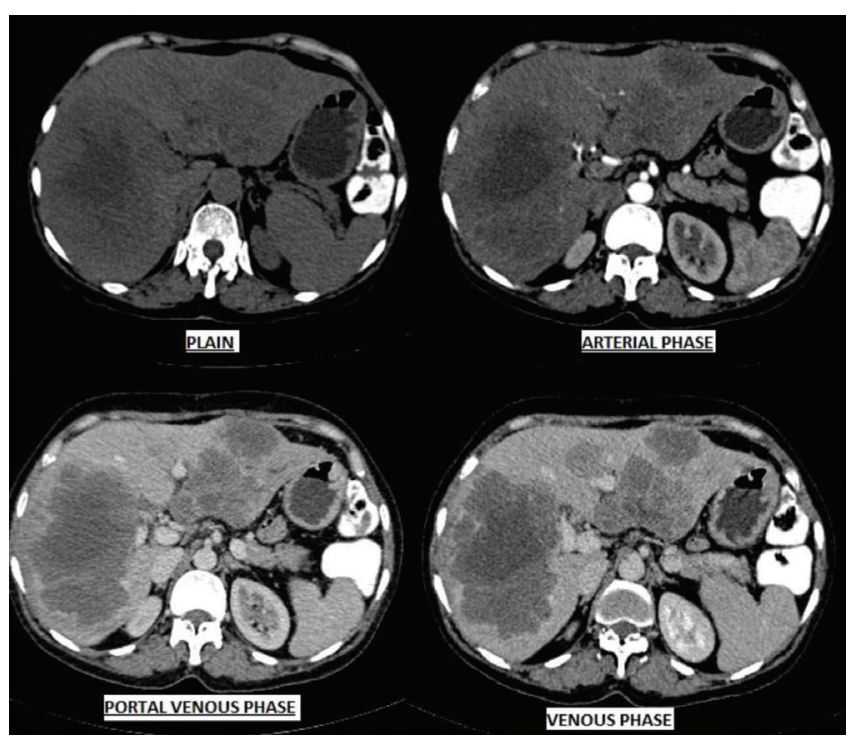

Figure-3: Axial CT scan images though liver in nonenhanced, arterial phase, portal venous and venous phase: hypovascular metastases. 


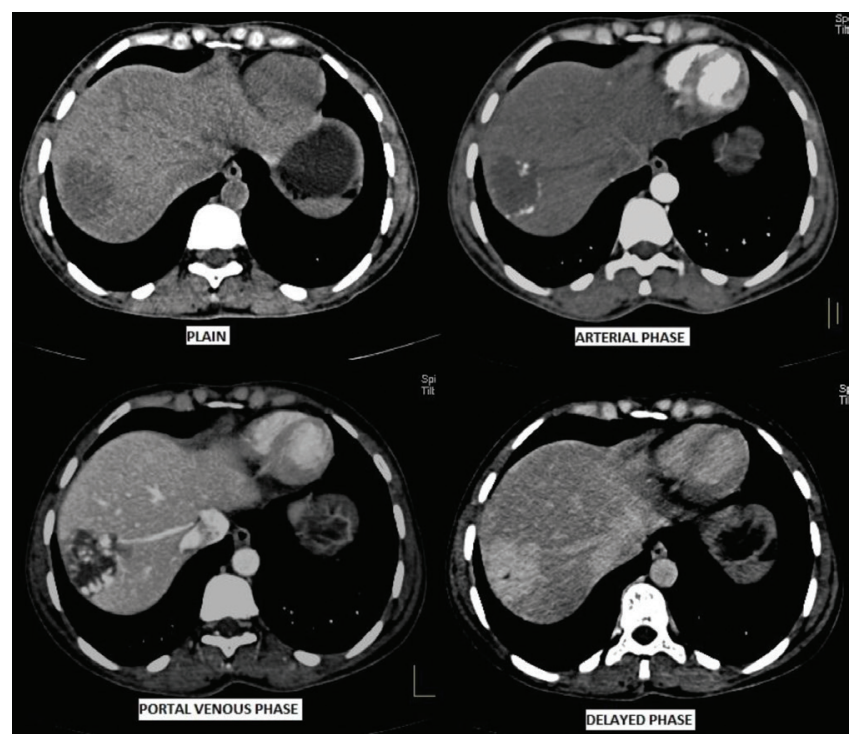

Figure-4: Axial CT scan images though liver in nonenhanced, arterial phase, portal venous and delayed phase: Haemangioma

$(66.67 \%)$ cases were focal lesions, 3 (20\%) were multifocal and $2(13.33 \%)$ were diffusely involving both lobes of liver. On NECT, 12 (80\%) cases showed isoattenuation to liver while 3 (20\%) cases showed low attenuation and $14 \quad(93.33 \%)$ hepatocellular carcinoma were heterogeneous and 1 (6.67\%) hepatocellular carcinoma was homogeneous; internal haemorrhage was seen in $4(26.67 \%)$ cases and necrotic areas were seen in $10(66.67 \%)$ cases. Calcification was not seen in any case of hepatocellular carcinoma. In contrast enhanced CT scan, 13 $(86.67 \%)$ cases showed heterogeneous hyper enhancement in the arterial phase; $8(53.33 \%)$ cases of hepatocellular carcinoma were hypoattenuating and 5 $(33.33 \%)$ cases were isoattenuating in portal venous phase, suggestive of early washout. In venous phase, 13 (86.67\%) cases of hepatocellular carcinoma were hypoattenuating and $2(13.33 \%)$ cases were heterogenous isoattenuating. Tumour thrombus in portal vein was seen in $6(40 \%)$ cases and bland thrombus was seen in 3 (20\%) cases.

In case of multiple lesions, size of the largest lesion was considered. Out of 41 cases of metastases, 29 (70.73\%) were small $(<3 \mathrm{~cm})$ and $12(29.27 \%)$ were large $(>3 \mathrm{~cm})$; multiple metastases were seen in $33(80.49 \%)$ cases and single metastasis was seen in $8(19.51 \%)$ cases. $32(78.05 \%)$ of metastases showed low attenuation, 8 (19.51\%) cases showed isoattenuation and 1 (2.44\%) case showed hyperattenuation. On nonenhanced CT scan, 18 (43.90\%) cases of hepatic metastases showed homogeneous appearance and 23 $(56.10 \%)$ cases showed heterogeneous appearance; calcification was seen in $2(4.88 \%)$ cases, internal haemorrhage was seen in $5(12.20 \%)$ cases and necrotic areas were seen in $30(73.17 \%)$ cases. On contrast enhanced CT scan, $39(95.12 \%)$ cases of metastases showed hypoattenuation in arterial phase and portal venous phase while, in the venous phase 12 (29.27\%) cases showed hypoattenuation and 27 $(65.85 \%)$ cases showed isoattenuation. One $(2.44 \%)$ case of hepatic metastases from carcinoid tumour showed hyperattenuating metastases in all the three phases, particularly in the arterial phase, consistent with hypervascular metastases.

Out of 7 cases of haemangioma, 3 $(42.86 \%)$ were smaller $(<3 \mathrm{~cm})$ and 4 (57.14\%) were larger $(>3 \mathrm{~cm}) ; 4(57.14 \%)$ cases showed single haemangioma and 3 (42.86\%) cases showed multiple haemangioma. On nonenhanced CT scan, 5 (71.43\%) cases of haemangioma showed low attenuation and 2 (28.57\%) showed isoattenuation; All 7 (100\%) cases of haemangioma showed homogeneous appearance. On contrast enhanced CT scan, 5 (71.43\%) cases of haemangioma, showed peripheral nodular enhancement in arterial phase and $6(85.71 \%)$ cases showed centripetal enhancement in portal venous and

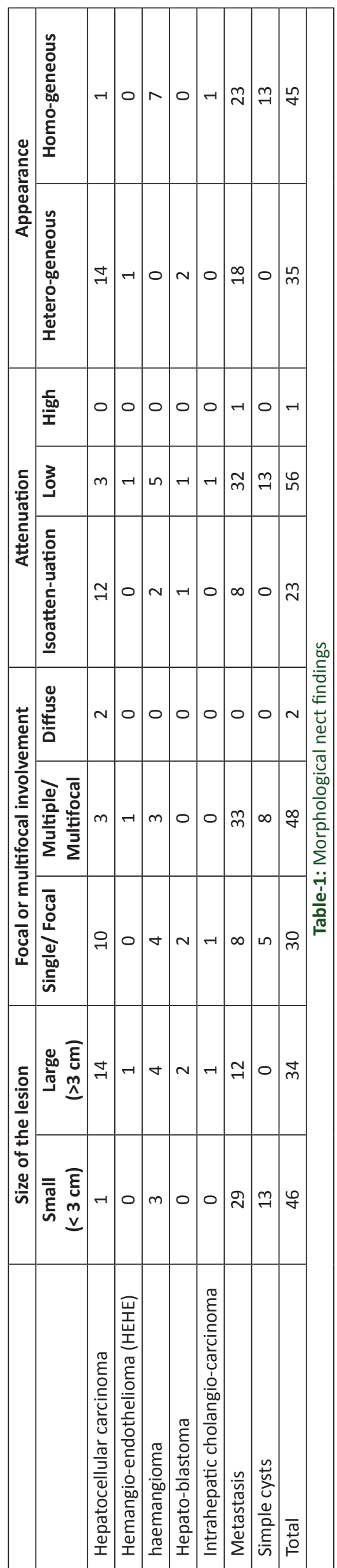


venous phases.

2 cases of hepatoblastoma and 1 case of intrahepatic cholangiocarcinoma were seen as single focal lesions, while, 1 case of hepatic epithelioid hemangioendothelioma (HEHE) was seen as multifocal lesion. Out of 13 cases of simple hepatic cysts, 5 (38.46\%) cases showed single and 8 (61.54\%) cases showed multiple simple cysts.

Low attenuation was seen in 1 case of hepatic epithelioid hemangioendothelioma ( $\mathrm{HEHE}), 1$ case of intrahepatic cholangiocarcinoma and all 13 cases of simple cysts. Out of 2 cases of hepatoblastoma, 1 case showed isoattenuation while 1 case showed low attenuation.

1 case of hepatic epithelioid hemangioendothelioma (HEHE) and 2 cases of hepatoblastoma showed heterogeneous appearance. 1 case of intrahepataic cholangiocarcinoma and all 13 cases of simple cysts showed homogeneous appearance. Using MDCT characteristic findings, out of 65 cases of adults with neoplastic lesions of liver including hepatocellular carcinoma, metastases, hemangioendothelioma, haemangioma and intrahepatic cholangiocarcinoma, sensitivity and specificity of MDCT for diagnosis of hepatocellular carcinoma was $86.7 \%$ and $98 \%$ respectively. Out of 80 cases, sensitivity and specificity of MDCT for diagnosis of hepatic metastases $97.5 \%$ and $92.3 \%$ respectively.

\section{DISCUSSION}

Imaging of the liver with the help of Multidetector CT scan is a common procedure in the current era. Multidetector CT permits scanning during multiple specific phases of intravenous contrast enhancement and the acquisition of very thin sections over a large area, allowing the creation of multiplanar reconstructions with high $\mathrm{z}$-axis resolution. A triple-pass technique, highlighting the arterial, parenchymal, and portal venous phases of enhancement, is recommended. ${ }^{1}$ Delayed phases are also taken as and when required particularly in the case of haemangioma and intrahepatic cholangiocarcinoma. The most common age group affected in our study was 41 to 70 years. We have classified the common neoplastic hepatic lesions into hepatocellular carcinoma, haemangioma, hepatic epithelioid hemangioendothelioma (HEHE), hepatoblastoma, intrahepatic cholangiocarcinoma, metastasis and simple cysts.

\section{Hepatocellular carcinoma}

hepatocellular carcinoma can have a variety of appearances. It most commonly presents as a large focal heterogeneous predominantly low attenuation mass which may have central necrosis. Calcification is uncommon in hepatocellular carcinoma. Hepatocellular carcinoma can also appear as a multifocal lesion or diffusely infiltrating lesion in both lobes of liver. In non-enhanced CT scan, hepatocellular carcinoma most commonly appears as a heterogeneous isoattenuating mass. Macroscopically, most large HCCs are expansile tumours with nodular morphology and surrounded by tumour capsules. Mosaic architecture is characteristic, defined by the presence of multiple internal tumour nodules separated by fibrous septations and areas of haemorrhage, necrosis, and occasionally fatty metamorphosis. ${ }^{2}$ Hepatocellular carcinoma shows typical heterogeneous hyper-enhancement in the arterial phase. In the portal venous phase, there is enhancement of the surrounding the liver parenchyma and the lesion appears hypoattenuated due to lack of portal venous supply. This characteristic is known as the washout of contrast. Sometimes, the washout is perceived only in the venous phase and delayed phase. Therefore, study of liver with four phases of contrast enhancement is recommended: non-contrast phase, late arterial phase, portal venous phase and delayed phase $^{3}$ (Figure 1,2).

\section{Hepatic metastasis}

Liver is one of the most common organ to be affected by metastasis. Hepatic metastases are much more common than primary hepatic neoplasms. The most common sites for primary include colon, breast, lung pancreas and stomach. ${ }^{4}$ Liver metastases are most commonly seen as multiple small lesions which show low attenuation on nonenhanced CT scan heterogeneous appearance. A study by Jungsung Choi showed that calcification can be seen hepatic metastases from mucinous gastrointestinal tumours. ${ }^{4}$ The lesion conspicuity will depend on differential enhancement between lesions and the adjacent liver parenchyma. A study by Jungsung choi showed that Hypervascular metastases including neuroendocrine tumours, melanoma, sarcoma, and renal cell carcinoma enhance more rapidly than normal liver parenchyma, resulting in greater conspicuity better detected in the hepatic arterial phase. ${ }^{4}$ Most of liver metastasis are hypovascular and are best imaged during the portal venous phase. During the equilibrium phase, lesions may become less conspicuous or completely obscured ${ }^{5}$ (Figure 3 ).

\section{Haemangioma}

Hepatic haemangioma are the most common benign hepatic tumour and are found incidentally in the rate of about 5 to $20 \%$ at routine radiologic examinations such as sonography and CT scan. ${ }^{6,7}$

The typical haemangioma appears as a hypoattenuating lesion on nonenhanced CT scan. Early peripheral nodular enhancement is seen in arterial phase. On the attenuation of the peripheral nodules is equal to that of the adjacent aorta. ${ }^{8}$ In venous phase, centripetal enhancement is seen with progresses to uniform filling in the delayed phase..$^{9,10}$

\section{Hepatic epithelioid hemangioendothelioma}

Hepatic epithelioid hemangioendothelioma (HEHE) is a rare vascular tumour of endothelial origin with low- to intermediate grade malignancy. ${ }^{11}$ It has been reported that most of HEHE cases are characterized by multiple lesions, solitary lesion only accounts for $13 \%-18 \%{ }^{12,13}$ Most of the lesions are peripheral, extending to the capsular margin. Capsular retraction adjacent to the mass is seen in fewer than $25 \%$ of patients. ${ }^{14}$ Tumour involvement can be widespread with extensive confluent masses and few traceable signs of portal or hepatic veins. After administration of IV contrast material, some tumour nodules display marginal enhancement during the arterial phase. ${ }^{14}$ On contrastenhanced scans, the tumour nodules may become isodense to liver parenchyma. The extent of involvement may be better defined on unenhanced images. ${ }^{15}$

\section{Hepatoblastoma}

Hepatoblastoma is the third most common intraabdominal malignant neoplasm in infants. ${ }^{16}$ Typically, hepatoblastoma 
is an aggressive tumour of large size at discovery (average, $10 \mathrm{~cm}$ ), and the prognosis is poor, particularly if the tumour has spread beyond planes that allow surgical resection. The radiologic findings in hepatoblastoma are those of a large mass, frequently with calcifications, a heterogeneous echogenic pattern on sonograms, and heterogeneous enhancement with hyperdense internal septa on CT scans. ${ }^{17}$

\section{Intrahepatic cholangiocarcinoma}

Intrahepatic cholangiocarcinoma is a carcinoma arising from any portion of the intrahepatic bile duct epithelium. It is the second most common primary intrahepatic malignancy after hepatocellular carcinoma and accounts for 10-20\% of all cases of intrahepatic malignancy. ${ }^{18,19}$ Mass-forming cholangiocarcinoma is characterized morphologically by a homogeneous mass with an irregular but well-defined margin and is frequently associated with dilatation of the biliary tree in the tumour periphery. ${ }^{20}$ The typical CT features of a mass-forming cholangiocarcinoma include homogeneous attenuation, irregular peripheral enhancement with gradual centripetal enhancement, capsular retraction, the presence of satellite nodules, and vascular encasement without the formation of a grossly visible tumour thrombus. ${ }^{20}$

\section{Simple hepatic cysts}

Hepatic cysts are common benign liver lesions that occur in $2 \%-7 \%$ of the population. These lesions may be isolated or multiple and vary from a few millimetres to several centimetres in diameter. ${ }^{21} \mathrm{~A}$ hepatic cyst appears as a homogeneous and hypoattenuating lesion on nonenhanced CT scans, with no enhancement of its wall or content after intravenous administration of contrast material. ${ }^{22}$

\section{CONCLUSION}

Multidetector CT scan with triple phase protocol of liver has become mainstay in the investigation of primary liver neoplasms and metastases. The most characteristic post contrast enhancement feature of hepatocellular carcinoma was heterogeneous hyperenhancement in arterial phase and washout in portal venous phase/venous phase which help to differentiate from hypovascular metastases. Hepatocellular carcinoma (HCC) typically shows heterogeneous hyperattenuation in arterial phase due to its hypervascular nature and supply by branches of hepatic artery. Metastases are usually hypovascular and typically appear hypodense on arterial and portal venous phase because the surrounding liver parenchyma enhances more than the lesion. Features like portal vein tumour thrombus or bland thrombus and arterioportal shunting were seen more commonly with hepatocellular carcinoma. MDCT proves to be "The Imaging Modality of choice" which is very good tool in characterizing neoplastic lesions of liver and is highly sensitive for detection of hepatocellular carcinoma and hepatic metastases.

\section{REFERENCES}

1. Ji, H., McTavish, J. D., Mortele, K. J., Wiesner, W., and Ros, P. R. Hepatic Imaging with Multidetector CT. RadioGraphics 2001;21(1):S71-S80.

2. Stevens, W. R., Gulino, S. P., Batts, K. P., Stephens, D. H., Johnson, C. D. Mosaic pattern of hepatocellular carcinoma: histologic basis for a characteristic CT appearance. Journal of computer assisted tomography 1996;20(3):337-342.

3. McEvoy, S. H., McCarthy, C. J., Lavelle, L. P., Moran, D. E., Cantwell, C. P., Skehan, S. J., Malone, D. E. Hepatocellular Carcinoma: Illustrated Guide to Systematic Radiologic Diagnosis and Staging According to Guidelines of the American Association for the Study of Liver Diseases. Radio Graphics 2013;33(6):1653-1668.

4. Choi, J. Imaging of Hepatic Metastases. Cancer Control 2006;13(1):6-12.

5. Sica, G. T., Ji, H., Ros, P. R. CT and MR Imaging of Hepatic Metastases. American Journal of Roentgenology 2000;174(3):691-698.

6. Ishak KG, Rabin L. Benign tumors of the liver. Med Clin North Am 1975; 59:995-1013.

7. Brannigan M, Burns PN, Wilson SR. Blood flow patterns in focal liver lesions at microbubble-enhanced US. RadioGraphics 2004; 24(3):921-935.

8. Soyer, P., Dufresne, A. C., Somveille, E., and Scherrer, A. Hepatic cavernous hemangioma: Appearance on T2-weighted fast spin-echo MR imaging with and without fat suppression. AJR Am J Roentgenol. 1997;168(2):461-5.

9. Nelson RC, Chezmar JL. Diagnostic approach to hepatic hemangiomas. Radiology 1990; 176(3):11-13.

10. Leslie DF, Johnson CD, MacCarty RL, Ward EM, Ilstrup DM, Harmsen WS. Single-pass CT of hepatic tumors: value of globular enhancement in distinguishing hemangiomas from hypervascular metastases. Am J Roentgenol 1995; 165(5):1403-1406.

11. Ishak, K. G., Sesterhenn, I. A., Goodman, Z. D., Rabin, L., and Stromeyer, F. W. Epithelioid hemangioendothelioma of the liver: a clinicopathologic and follow-up study of 32 cases. Hum Pathol. 1984;15(9):839-52.

12. Makhlouf, H. R., Ishak, K. G., and Goodman, Z. D. Epithelioid hemangioendothelioma of the liver: A clinicopathologic study of 137 cases. Cancer 1999;85:2$\mathrm{T}$.

13. Mehrabi, A., Kashfi, A., Fonouni, H., Schemmer, P., Schmied, B. M., Hallscheidt, P., Schmidt, J. Primary malignant hepatic epithelioid hemangioendothelioma: A comprehensive review of the literature with emphasis on the surgical therapy. Cancer. 2006;107(9):2108-21.

14. Miller, W. J., Dodd, G. D., Federle, M. P., and Baron, R. L. (1992). Epithelioid hemangioendothelioma of the liver: Imaging findings with pathologic correlation. In American Journal of Roentgenology. https://doi. org/10.2214/ajr.159.1.1302463.

15. Radin, D. R., Craig,J. R., Colletti, P. M., Ralls, P.W., and Halls, J. M. Hepatic epithelioid hemangioendothelioma. Radiology 1988;169(1):145-148.

16. Amendola, M. A., Blane, C. E., Amendola, B. E., and Glazer, G. M. CT findings in hepatoblastoma. Journal of Computer Assisted Tomography 1984;8(6):11051109.

17. Stoupis, C., and Ros, P. R. (1993). Imaging findings in hepatoblastoma associated with Gardner's syndrome. American Journal of Roentgenology. https://doi. 
org/10.2214/ajr.161.3.8394642

18. Ros, P. R., Buck, J. L., Goodman, Z. D., Ros, A. M., and Olmsted, W. W. Intrahepatic cholangiocarcinoma: radiologic-pathologic correlation. Radiology 1988;167(3), 689-693.

19. Choi, B. I., Park, J. H., Kim, Y. I., Yu, E. S., Kim, S. H., Kim, W. H., Han, M. C. Peripheral cholangiocarcinoma and clonorchiasis: CT findings. Radiology, 1988;169(1): 149-153.

20. Chung, Y. E., Kim, M.-J., Park, Y. N., Choi, J.-Y., Pyo, J. Y., Kim, Y. C. Choi, S. Y. Varying Appearances of Cholangiocarcinoma: Radiologic-Pathologic Correlation. RadioGraphics 2009;29(3):683-700.

21. Horton, K. M., Bluemke, D. A., Hruban, R. H., Soyer, P., and Fishman, E. K. CT and MR Imaging of Benign Hepatic and Biliary Tumors. RadioGraphics, 1999; 19(2): 431-451.

22. Mathieu, D., Vilgrain, V., Mahfouz, A. E., Anglade, M. C., Vullierme, M. P., and Denys, A. Benign liver tumors. Magnetic Resonance Imaging Clinics of North America 1997;5(2):255-288.

Source of Support: Nil; Conflict of Interest: None

Submitted: 14-08-2018; Accepted: 10-09-2018; Published online: 01-10-2018 